\title{
Biological Function of CIRC-RNA and Its Role in Tumorigenesis Jialin Guo ${ }^{1, a}$, Xiaozhi Yang ${ }^{1, b}$ \\ ${ }^{1}$ Luohe Medical College, Luohe, Henan, 462000 \\ ${ }^{a}$ email, ${ }^{b}$ email
}

\section{Keywords: Circular RNA, Micro RNA Sponge, Tumor, Occurrence}

\begin{abstract}
Over the years, with advances in RNA research, researchers have found not only the large number of circular RNAs found in many organisms, but also their important biological functions. Cyclic RNA is derived from introns or exons, can act as a micro RNA sponge, but also with the protein combination to participate in the regulation of gene expression and affect the function of the protein, in addition to individual circular RNA even can encode the protein. More importantly, cyclic RNA in tumor (such as: gastric cancer, liver cancer, colorectal cancer, breast cancer, cervical cancer and ovarian cancer, etc.) play an important role in the process of development and regulation. Therefore, the circular RNA is expected to become a tumor marker and a new target for treatment.
\end{abstract}

\section{Introduction}

Circular RNA (circular RNA) is a class of closed-loop RNA molecules that are widely present in the transcriptional group. Participate in a variety of biological activities of biological regulation. CircRNA is not easily degraded by nuclease, more stable than linear RNA, and may be an ideal diagnostic marker for disease. With the deep research of transcription group and the development of RNA sequencing technology, researchers have detected a large number of circRNA in vivo. Their interactions with proteins can affect cells

Cell cycle senescence is apoptosis and other basic life activities. In addition, circRNA also with gastric cancer, colorectal cancer, breast cancer, cholangiocarcinoma, pancreatic cancer and ovarian cancer and other tumors have a direct or indirect development. This article aims at domestic and foreign

Recent advances in the research of circiRNAs are reviewed in this paper, including species, biosynthesis, features, functions and their relationship with tumor.

\section{Classification of Circular RNA}

CircRNA is a class of RNA molecules that have been neglected for a long time and have important biological functions. As early as 1976, the first circRNA was found in the virus. Subsequently, the researchers found in a variety of different organisms more and more circRNA, including archaea circRNA, mouse circRNA and human circRNA and so on. RNA sequencing, single-cell RNA sequencing and other technologies, researchers have found a variety of organisms in a considerable number of circRNA. There are three main types of circiRNAs: circular RNA (cRNA), exon sequence (exon), exon sequence (exon), exon sequence (exon) and exon sequence (exon) -interron circular RNA, EIciRNA). The vast majority of circRNAs in humans and mice come from coding genes.

\section{Loop RNA Biosynthesis}

In single-celled organisms, circRNA is mainly derived from the self-splicing of pre-ribosomal RNA gene transcripts, but in archaea it is transcribed by protein-coding genes. CircRNA in animals is mainly from the splice in the same exon 5 'end and downstream 3' sequence from the cyclization. Three types of circiRNAs were generated in different ways. In the formation of ecircRNA, the most common way is the tail-tail splicing. The 2 '-hydroxyl group upstream of the bifurcation point of the 
cyclized exon attacked the downstream 5' splice site to form an intermediate product of the Y-type structure. Secondly, the exon One step of the newly formed 3 '- hydroxyl terminal attack upstream of the $3^{\prime}$ splice site, the final release of a circular RNA. In the formation of ecircRNA, the retention of intron sequences will produce EIciRNA. At the tail-splicing, the formation of circRNA-dependent flanking sequences, including repetitive or non-repeating elements, facilitates the efficient formation of circRNA. In addition, the splice sites should be close to each other to promote tail splicing. This step, despite its simple mechanism, is highly selective, and these repeats can radically alter the efficiency of the production of circRNA. The longer exons appear to have higher cyclization efficiency, and the processing of the 3 'end is required for cyclization, suggesting that the formation of circRNA may be at the post-transcriptional stage. However, during the formation of ciRNAs, critical sequences (such as certain intron sequences) are required for cyclization to proceed normally. Some researchers found that ciRNA formation process there are some common conserved sequence, such as: shear site near the existence of a $7 \mathrm{nt}$ rich GU base structure, there is a branch near the $11 \mathrm{nt}$ rich in $\mathrm{C}$ Base structure.

Indeed, these length-defining key RNA elements increase the efficiency of ciRNA formation, while other cis-acting elements may further promote the formation of the circRNA. Interestingly, Zhang et al. Found that the same gene locus can generate multiple circiRNAs. For example, human CAMSAP1 gene can produce at least seven different ecircRNAs. They call this phenomenon Alternative circularization (AC). In theory, this feature will make the regulation of gene expression in the circRNA more flexible and convenient.

\section{Biological Characteristics of Circular RNA}

Most of the circRNA from the exon of the tail tail splicing, and stable presence in the cytoplasm. Cytoplasm of the circRNA most of them have a half-life of more than $48 \mathrm{~h}$, while the average half-life of mRNA is only 10 . The stability of circiRNAs may be due to their lack of mRNA-like 5 ? Cap and 3? Tail structure, so you can avoid the removal of adenosine, cap-off reaction and mRNA-related normal degradation reaction. Some circRNAs are specifically expressed in different tissues and at different stages of development. The conserved nucleotide sequence of the circRNA was significantly increased, indicating that the circRNA can compete with other RNA or microRNA (miRNA) for binding to RNA binding protein (RBP). Competitive endogenous RNA (Competitive Endogenous RNA) competes with the miRNA by its own miRNA response element (MRE), compared to other miRNA-containing ceRNAs (pseudogene transcripts, lncRNAs and mRNAs), The stability of circRNA because of its special structure makes it an advantage ceRNA, plays an important role in the balance of ceRNA network.

Most human circRNAs are only expressed in a few cell types and have very low levels of expression, and their cell specificity is not as strong as the corresponding mRNA. Although most circRNAs contain protein-coding sequences, ribosomal analysis does not prove that they can direct protein synthesis. It was found that 635 kinds of mouse circRNA and $20 \%$ of human circRNA is orthologous, but these homologous sequences of the conserved sequence of circa is homologous linear exon is not high. One of the major roles of the circiRNAs in the cytoplasm derived from the tail-tail splicing is as a miRNA sponge, and the function of the abundant ciRNAs in the nucleus is different from the cytoplasmic circRNA. A series of experiments have shown that ciRNA is associated with certain bases

CiRNAs that are abundant in the nucleus may participate in cis regulation of gene expression. CiRNA in humans

Like cells, but the evolutionary conservatism was low. In addition, the researchers also found that some ciRNA species-specific. Thus, different ciRNAs can be identified using the same or similarities specific for different tissues and species. 


\section{Circular RNA}

The biological function of biological macromolecules is closely related to its structure and properties. Recent studies on the functions of circiRNAs have shown that they play a major role in miRNA sponges, transcriptional regulation, binding proteins and translation.

The miRNA sponge is one of the most hot spots in the research of circRNA, and it may be a common function. Among the circRNAs that can act as miRNA sponges, the most typical is ciRS-7 (Circular RNA sponge for miR-7). It contains more than 70 conserved miRNA-binding sites and is likely to be associated with the Argonaute, AGO-dependent miR-7. The circRNA strongly inhibits miR-7 activity, thereby increasing the level of the miR-7 target. Another circiRNA, a miRNA sponge, is a testis-specific transcript of the sex-determining region Y (Sry), which contains the 16 conserved binding sites of miR-138.

The newly discovered heart-related circular RNA (HRCR), a miR-223 sponge, inhibits the activity of miR-223, resulting in increased expression of miR-223 downstream genes, which ultimately slows cardiac hypertrophy and heart failure . MiRNA sponge is the main role of circRNA as a ceRNA. In vivo, ceRNA network complex and fine, any small disorder will change the gene expression, leading to tumorigenesis and circRNA as one of the important members of the maintenance of ceRNA network balance essential; In addition, the study miRNA miRNA sponge May explain some of the molecular mechanisms of tumorigenesis.

Mouse formin (Formin) is essential for limb development, its gene fmn can be transcribed to form a circRNA. In the tail-splicing process involved in fmn gene coding sequence upstream of a splicing receptor, if the knockout mouse fmn gene in this splicing receptor, will not detect the circRNA, mouse limb normal development, but there are incomplete Infiltration of renal hypoplasia of the phenotype. This indicates that the splice acceptor of the circRNA is involved in the transcription and translation of the mouse gene. If it is missing, it may cause some diseases. In combination with biochemistry, cell function testing and bioinformatics analysis, the authors found that the circadian RNA of antisense to the cerebellar degeneration-related protein 1 transcript (CDR1as) has the function of down-regulating miR-7, While miR-7 has complete sequence conservatism in both $\mathrm{C}$. elegans and humans. This also indicates that circRNA is an important post-transcriptional regulator.

CircRNA can stably bind to AGO protein and RNA polymerase II. In addition, they can be combined with a variety of RBP RBP scaffolding. The circRNA may also serve as a target sequence element, utilizing its complementary sequence to simultaneously bind RBP, RNA or DNA. More recently, researchers have found that overexpression of Circovirus-3 (O3) in the heart tissue of adult mice and elderly patients with heart disease has been shown to be overexpressed; further studies have shown that circ-Foxo3 And hypoxia inducible factor $1 \alpha$ (HIF $1 \alpha)$ and focal adhesion kinase (FAK). Because HIF1 $\alpha$ and FAK have the role of inhibiting cell senescence, when the expression of circ-Foxo3 in the cytoplasm increases, these proteins are captured, their function can not play, will lead to accelerated cell aging process. Thus, abnormal high expression of circ-Foxo3 can promote cell senescence, and silencing of the circRNA can slow cell senescence.

In mammalian cells, exogenous circulatory RNA encoding proteins has been detected, such as RNA derived from Hepatitis D virus (HDV). HDV is a satellite virus of hepatitis B virus (HBV). The circadian RNA of HDV expresses the pathogenic viral protein. If the circRNA contains an internal ribosome entry site, it is possible to direct protein synthesis, and the eukaryotic ribosomal $40 \mathrm{~S}$ small subunit can enter the internal ribosome entry site of some circins to direct protein synthesis. Experiments show that if the circRNA contains a base that is a multiple of 3, the circulatory RNA of the encoded protein is read along the reading frame loop, thereby translating a repeating polypeptide sequence. However, so far, there is no evidence that eukaryotic endogenous circiRNAs can direct protein synthesis, and no translationally generated polypeptides have been found. However, many circRNAs contain the initiation codon, and some even contain typical AUG promoters in linear transcripts, so exploring endogenous circiRNAs as transcripts remains an active research direction. 
The above studies have shown that HDV is the only protein that can encode the circRNA. Although most of the circRNAs are from coding regions and even contain initiation codons, they are non-coding RNAs.

\section{Circular RNA And Tumor}

In recent years, more and more studies have shown that circRNA through direct or indirect role in tumorigenesis and development plays an important role. For example, some studies have found that the total cell cycle of colorectal cancer and the degree of cancer metastasis associated with the total; the first time in our group found, Has_circ_002059 and gastric cancer patients with a variety of clinical pathology is closely related to the diagnosis of gastric cancer is expected to become a new symbol . To this end, in-depth understanding of the role of circRNA in tumorigenesis and development, will help reveal the mechanism of tumor, tumor diagnosis and treatment to find a new method.

MiRNA is a kind of regulatory non-coding RNA which is prevalent in organism. They typically regulate gene expression at post-transcriptional levels by degrading or inhibiting the target mRNA. MiRNA can play a carcinogenic or anti-cancer effect. Some circRNAs contain miRNA binding sites, can regulate the biological function of miRNA, and thus indirectly involved in the occurrence and development of cancer process. In the regulatory network of non-coding RNA, the combination and competition of circRNA and miRNA provides a new idea for us to understand the process of cancer development and development. As described above, ciRS-7 binds miR-7, thereby inhibiting miR-7 from exerting its biological function.

In gastric cancer cells, miR-7 can inhibit insulin-like growth factor receptor (Insulin-like growth factor 1 receptor, IGF1R. The reduction of IGF1R expression increased the expression of E-cadherin, partially reversing the epithelial-mesenchymal transition, resulting in cancer cell metastasis and proliferation blocked, delaying the growth and invasion of cancer cells in the process. Therefore, down-regulation of miR-7 by ciRS-7 results in the release of IGF1R proto-oncogene and induces migration and proliferation of cancer cells. In colorectal cancer, miR-7 has tumor suppressive effect. It can inhibit the expression of oncogene yin yang1. The oncogenes act by inhibiting P53, modulating the downstream P15 effector, the caspase cascade and proto-oncogene c-jun; and activating Wnt by activating beta-catenin, survivin and fibroblast growth factor-4 signal path. Therefore, miR-7 can inhibit the proliferation of colorectal cancer cells and induce apoptosis. In addition to gastrointestinal tumors, miR-7 and other cancer also has a relationship. MiR-7 inhibits Her-2-regulated breast cancer and partially reverses trastuzumab resistance by inhibiting multiple tumor-related signaling pathways. In addition, miR-7 can increase the sensitivity of non-small cell lung cancer cells to paclitaxel. A cervical cancer study found that miR-7 at the level of transcription and translation by focal adhesion kinase as a target to inhibit cervical cancer metastasis and invasion.

From the above, miR-7 regulates a variety of signaling pathways closely related to tumor, and ciRS-7 can indirectly affect the occurrence and development of tumor through miR-7 binding.

In addition to ciRS-7, there are some other circRNA on tumor occurrence and development have a significant impact. Sry is a testicular-specific circular transcript expressed by a male sex-determining gene. As a miRNA sponge, sry regulates its biological function by binding to the 16 conserved sites on miR-138. In cholangiocarcinoma cells, sry inhibited the expression of miR-138, resulting in increased mRNA and protein expression of member $\mathrm{C}$ of the Ras homologous gene family. As a result, the RhoC / p-ERK / MMP-2 / MMP-9 pathway, Thereby promoting the proliferation, migration and invasion of cholangiocarcinoma cells. In colorectal cancer, miR-138 directly targets Twist2, a member of the basic helix-loop-helix family, hampering its function and ultimately suppressing the migration and invasion of colorectal cancer cells.

Significant down-regulation of miR-138 in CLL was associated with inhibition of CD95-regulated apoptosis. CirciRNA is a kind of regulatory molecule which has been paid more attention to by researchers. Most researches about its functions have been found to interact with downstream targets, such as ciRS-7 and sry, respectively, and their downstream targets miR-7 and 
miR- 138 \& lt; RTI \& gt; Identification of other circiRNAs as miRNA sponges has also been ongoing, such as the recently discovered circular RNA-HRCR as miR-223 sponge. Since thousands of unknown circulatory molecules are identified in different species, it is reasonable to believe that more and more interactions between the circiRNAs and corresponding miRNAs will occur and that their involvement in a wider variety of cancers Regulation process.

From the discovery of circRNA so far, its relationship with the tumor has been a hotspot. Researchers hope to find the pathogenesis of the tumor from the circRNA, as well as new methods of diagnosis and treatment of tumors. The expression level of cir-ITCH was significantly down-regulated in esophageal squamous cell carcinoma compared with that in the adjacent non-cancerous tissues, and the expression level of Has_circ_002059, Has_circ_0001649 and Has_circ_104912 were significantly lower in colorectal cancer tissues, $\overline{\mathrm{HCC}}$ and laryngeal squamous cell carcinoma, while the expression level of Has_circ_100855 was significantly up-regulated in laryngeal squamous cell carcinoma. The abnormal expression of these circRNAs in cancerous tissues indicates that circRNA is involved in the development and progression of tumors.

\section{Conclusion}

With the deepening of the study of the circRNA, we understand it more widely. Although circRNA was discovered decades ago, it has received much attention in recent years, and more and more important discoveries have been made about it. The results showed that Has_circ_002059 has a potential role as a new type of gastric cancer, and has been used as a new biomarker for cancer diagnosis. It has been reported that Has_circ_002059 is a new type of gastric cancer, which has the characteristics of structural particularity and biological stability. Marker possibilities; Lukiw et al

Studies have shown that the level of CDRlas expression in patients with Alzheimer's disease is reduced. On the other hand, the search for tumor diagnostic markers, the collection of body fluids (saliva, blood, etc.) trauma is small, easy to obtain and other characteristics, has been the ideal choice for researchers. Yan et al found that exosomes in the circRNA (Exosome circRNA, exo-circRNA)

Rich and stable in nature, and the expression of a variety of serum exo-circRNA in cancer patients and healthy people have significant differences, according to the patients and the normal distinction. Although circRNA is involved in the regulation of gene expression and the development and progression of tumor, most of the circRNAs have been found to function indirectly by regulating miRNAs. There is a competitive relationship and a synergetic relationship between circiRNA and miRNA, and the balance between circiRNA and miRNA is vital to the organism. We can use the circRNA as a target, to control miRNA, and then regulate a series of life activities. Therefore, clinically, it is possible for the targeted treatment of certain tumors for the circRNA. All of these have great research value and application prospect.

\section{References}

[1] Sanger HL, Klotz G, Riesner D, et al. Viroids aresingle-stranded covalently closed circular RNA molecules existing as highly base-paired rod-likestructures. Proc Natl Acad Sci USA, 1976, 73(11):3852-3856.

[2] Kjems J, Garrett RA. Novel splicing mechanismfor the ribosomal RNA intron in the archae bacterium desulfurococcus mobilis. Cell, 1988, 54(5): 693-703.

[3] Danan M, Schwartz S, Edelheit S, et al.Transcriptome-wide discovery of circular RNAsin Archaea. Nucleic Acids Res, 2012, 40(7):3131-3142.

[4] Capel B, Swain A, Nicolis S, et al. Circular transcripts of the testis-determining gene Sry inadult mouse testis. Cell, 1993, 73(5): 1019-1030.

[5] Burd CE, Jeck WR, Liu Y, et al. Expression oflinear and novel circular forms of anINK4/ARF-associated non-coding RNA correlates with atherosclerosis risk. PLoS Genet, 2010, 6(12): e1001233. 
[6] Memczak S, Jens M, Elefsinioti A, et al. Circular RNAs are a large class of animal RNAs with regulatory potency. Nature, 2013, 495(7441):333-338. 